\title{
DEVELOPMENT OF EXUDATIVE AGE-RELATED MACULAR DEGENERATION AFTER CATARACT SURGERY
}

\author{
AYALA POLLACK, ARIE MARCOVICH, AMIR BUKELMAN, MIRIAM ZALISH and \\ MOSHE OLIVER \\ Rehovot, Israel
}

\begin{abstract}
SUMMARY
The macular status of patients with findings consistent with early dry age-related macular degeneration (AMD) who underwent an extracapsular cataract extraction with intraocular lens implantation was evaluated by fundoscopy and fluorescein angiography pre-operatively and during the first post-operative year. Five patients who developed the exudative form of AMD, and who represent the problems arising when treating patients with AMD and cataract, are described. Patients who received laser photocoagulation responded with recurrent choroidal neovascularisation. We suggest that patients with signs of age-related changes scheduled for cataract surgery should undergo a thorough pre- and post-operative assessment of their retinal status.
\end{abstract}

Cataract surgery may be followed by deterioration of retinopathic changes. ${ }^{1}$ Recently aggravation of diabetic retinopathy has been described. ${ }^{2-8}$ However, this is not the only retinal condition that may worsen after surgery. We observed deterioration of the exudative form of age-related macular degeneration (AMD) during the first year after cataract surgery. ${ }^{1,9}$ The purpose of this study is to call attention to this possibility and to describe the post-operative maculopathic course of five cases.

Case 1

\section{CASE REPORTS}

A 76-year-old man was followed for glaucoma and AMD. On admission, fundoscopy and fluorescein angiography of the right eye disclosed retinal pigment epithelial (RPE) defects that corresponded to drusen and to the hypopigmented area, without evidence of choroidal neovascularisation (CNV).

Correspondence to: A. Pollack, MD, Department of Ophthalmology, Kaplan Hospital, 76100 Rehovot, Israel. Tel: 972-8-441351. Fax: 972-8-441-377
The cup/disc ratio was 0.5 . Fundoscopy and angiography of the left eye showed drusen. The cup/disc ratio was 0.6 .

Eight years later he was scheduled for extracapsular cataract extraction (ECCE) with intraocular lens (IOL) implantation in the right eye. Evaluation prior to and immediately after this operation revealed unchanged macular status in both eyes. Following the operation, there was no significant intraocular inflammation and vision in the operated eye improved to $20 / 40$ with unchanged maculopathy (Fig. 1a). The subsequent follow-up was unremarkable until 10 months after surgery, when he complained of a sudden loss of vision in the right eye. Visual acuity in that eye was finger counting before the eye and 20/30 in the left. Fundoscopy and angiography of the right eye were consistent with CNV (Fig. 1b). The left eye showed stable maculopathy (Fig. 1c).

Case 2

A 73-year-old man with a 12 year history of orally controlled diabetes mellitus was followed since 1982. On admission fundoscopy revealed drusen in both eyes but no diabetic retinopathy. Fluorescein angiography showed RPE defects corresponding to drusen, with no evidence of $\mathrm{CNV}$ and/or diabetic retinopathy (Fig. 2a).

Ten years after admission the patient was scheduled for cataract surgery in the right eye. Fluorescein angiography prior to this operation showed no diabetic retinopathy and stable RPE defects in both eyes (Fig. 2b,c). The patient underwent an uneventful ECCE with IOL implantation, with no remarkable intraocular inflammation post-operatively. One month after surgery, vision in the operated right eye was 20/70. Fundal examination revealed a minute intraretinal haemorrhage superior to the fovea and a flame-shaped haemorrhage on the temporal side of 


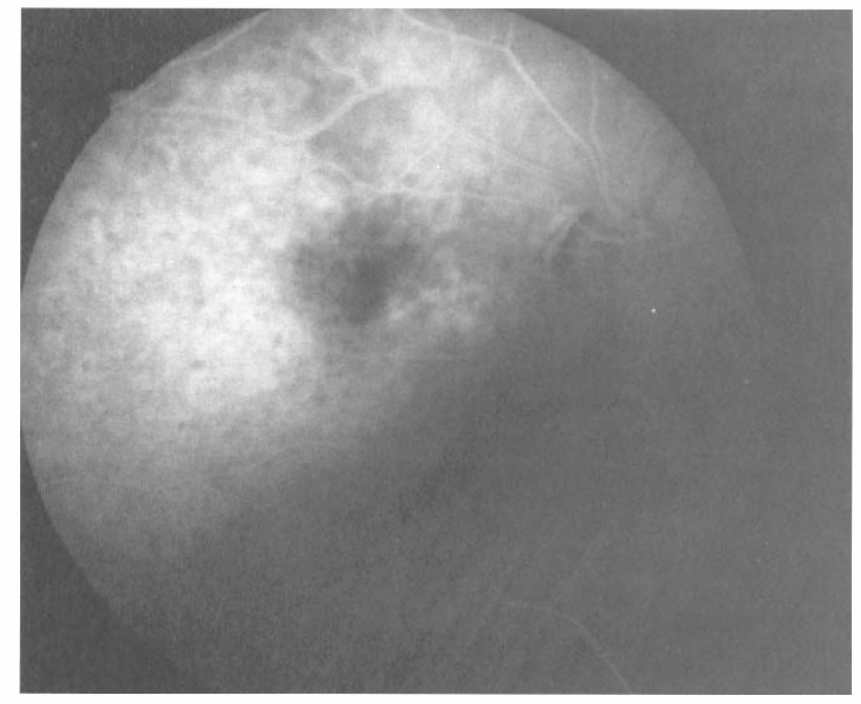

(a)

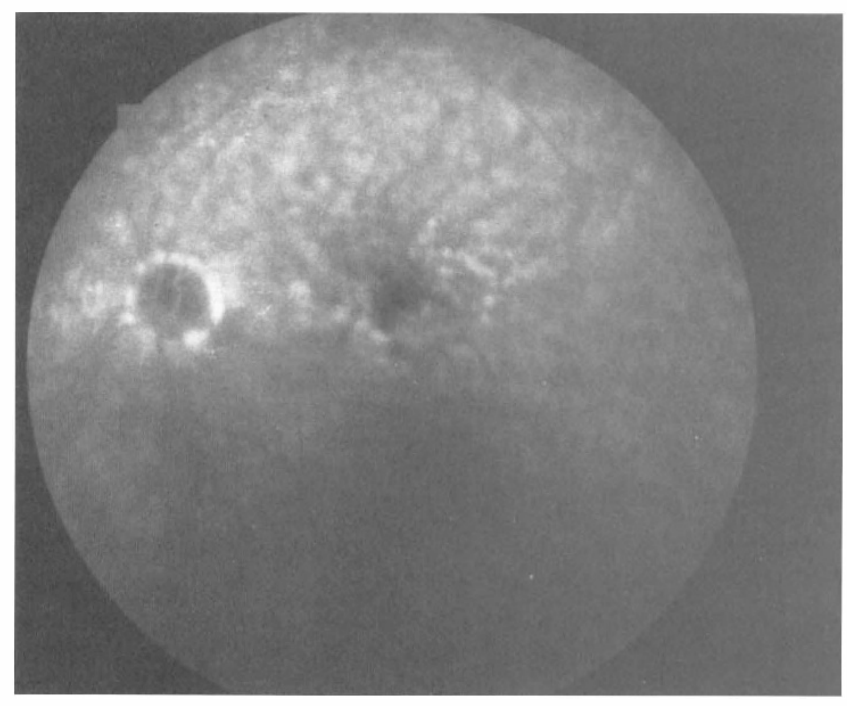

(c)

the optic disc. Angiography showed two foci of blocked fluorescence corresponding to the haemorrhages and RPE defects correlated with drusen (Fig. 3a). Three months later the vision in the right eye had deteriorated to 20/300 and fundoscopy revealed subretinal haemorrhage suspected for $\mathrm{CNV}$ and retinal mild diabetic retinopathy. Fluorescein angiography disclosed vascular abnormalities consistent with diabetic retinopathy and $\mathrm{CNV}$ with late leakage (Fig. 3b,c). The maculopathy of the left eye remained stable. The patient received argon laser photocoagulation, but subsequently developed recurrent $\mathrm{CNV}$ and his vision decreased to finger counting before the eye.

\section{Case 3}

A 66-year-old man was referred for cataract surgery. He had a history of diet-controlled diabetes mellitus and chronic lung disease. Both eyes showed visual

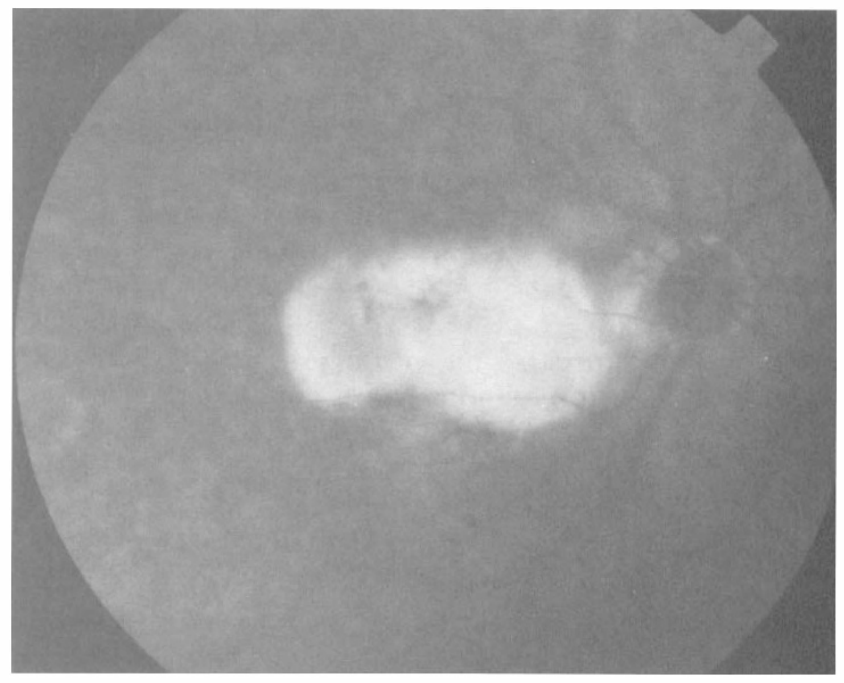

(b)

Fig. 1. Case 1. Follow-up after surgery of the right eye. (a) The immediate post-operative angiogram shows retinal pigment epithelial (RPE) defects corresponding to drusen and to the area of hypopigmentation, without leakage suspected for choroidal neovascularisation $(C N V)$. (b) and (c) Ten months after surgery. (b) The right eye shows leakage from $C N V$. (c) The left eye shows stable RPE defects corresponding to drusen.

acuity of 20/100 and progressive nuclear sclerosis cataract. Fundoscopy and angiography were consistent with drusen. No leakage suspected for $\mathrm{CNV}$ was seen. The patient underwent uneventful ECCE with IOL implantation in the left eye with no remarkable post-operative intraocular inflammation and his vision improved to 20/30. Seven months after surgery the patient complained of decreased vision in the operated eye. The non-operated eye remained unchanged (Fig. 4a,b). Vision in the operated eye had declined to 20/50. Fundoscopy revealed a small RPE detachment and angiography demonstrated leakage from CNV (Fig. 4c,d). The patient received argon laser photocoagulation and his final vision was $20 / 400$.

\section{Case 4}

An 83-year-old man was referred for cataract extraction. His vision was $20 / 400$ in the right eye 


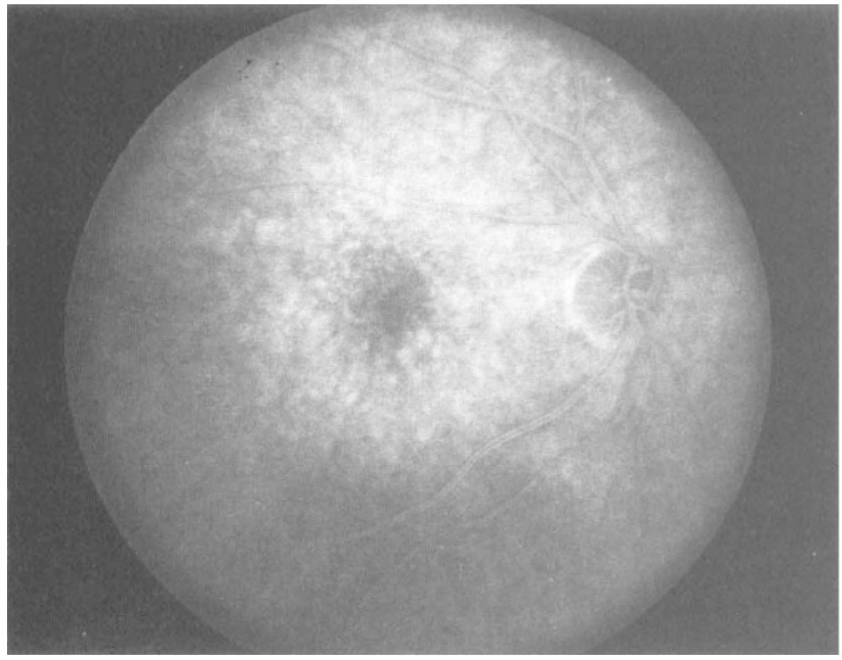

(a)

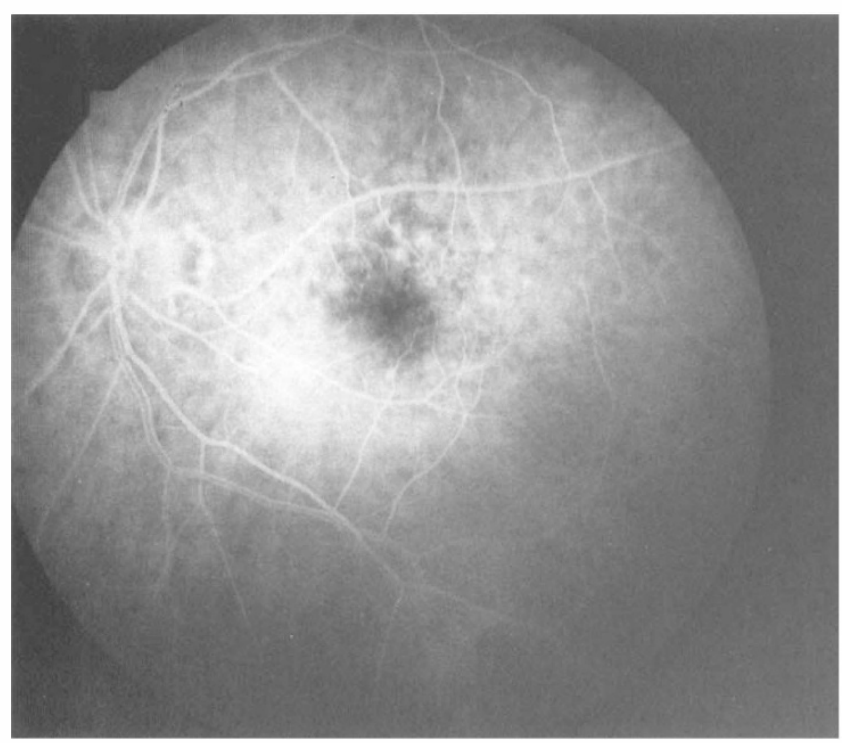

and $20 / 40$ in the left. Biomicroscopy revealed progressive nuclear sclerosis cataract in the right eye and moderate cataract in the left. The fundus of the right eye could not be clearly seen because of the cataract. Ultrasonography disclosed a flat retina. Fundoscopy of the left eye showed drusen. The patient underwent uneventful ECCE with IOL implantation in the right eye with no remarkable intraocular inflammation. One week post-operatively the vision had improved to 20/50, and fundoscopy and angiography were consistent with drusen (Fig. 5a). One month later the patient complained of metamorphopsia and decreased vision in the operated eye, the acuity of which had indeed declined to 20/400. Fundoscopy of that eye revealed RPE detachment. Angiography showed RPE detachment with suspected occult CNV in the operated eye (Fig. 5b), and RPE defects corresponding to drusen in the fellow eye (Fig. 5c). A grid pattern of argon laser photocoagulation was applied in the operated

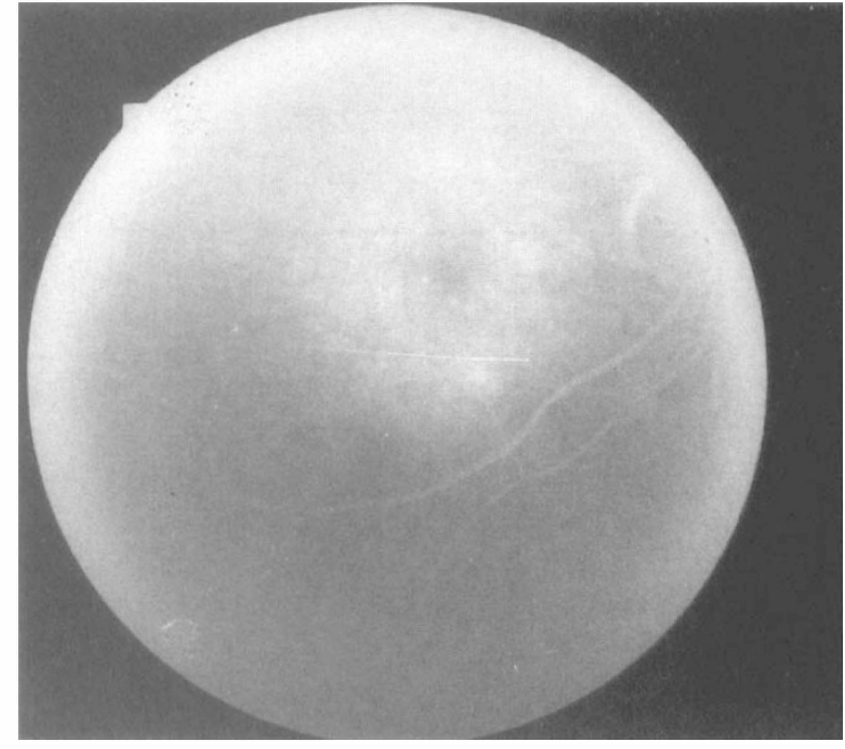

(b)

Fig. 2. Case 2. (a) Fluorescein angiography 7 years prior to surgery of the right eye shows RPE defects corresponding to drusen with no leakage suspected for CNV. (b) Immediate pre-operative angiogram of the right eye demonstrates unchanged maculopathy (compare with (a)). (c) Angiogram of the left eye taken at the same time as (b) shows RPE defects corresponding to drusen.

eye, after which the detachment subsided and the vision improved to $20 / 200$. Two months later he developed recurrent CNV.

\section{Case 5}

A 76-year-old woman who had been followed for 4 years because of AMD in the left eye was scheduled for cataract surgery. Her medical history revealed drug-controlled hypertension; her visual acuity was $20 / 100$ in the right eye due to cataract and finger counting at $1 \mathrm{~m}$ in the left due to AMD. Preoperative fluorescein angiography of the right eye showed RPE defects that appeared unchanged from previous angiograms without leakage suspected for CNV (Fig. 6a,b). The patient underwent uneventful ECCE with IOL implantation in the right eye with no remarkable post-operative intraocular inflammation. The post-operative visual acuity in the right eye was $20 / 70$ with mild opacification of the posterior capsule and stable maculopathy. Eight months after 


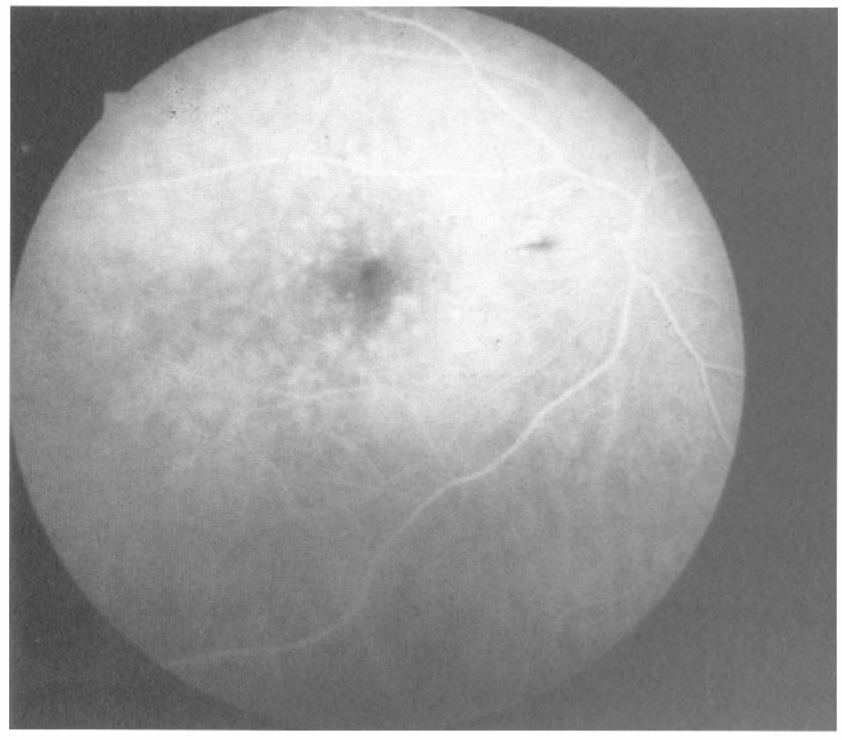

(a)

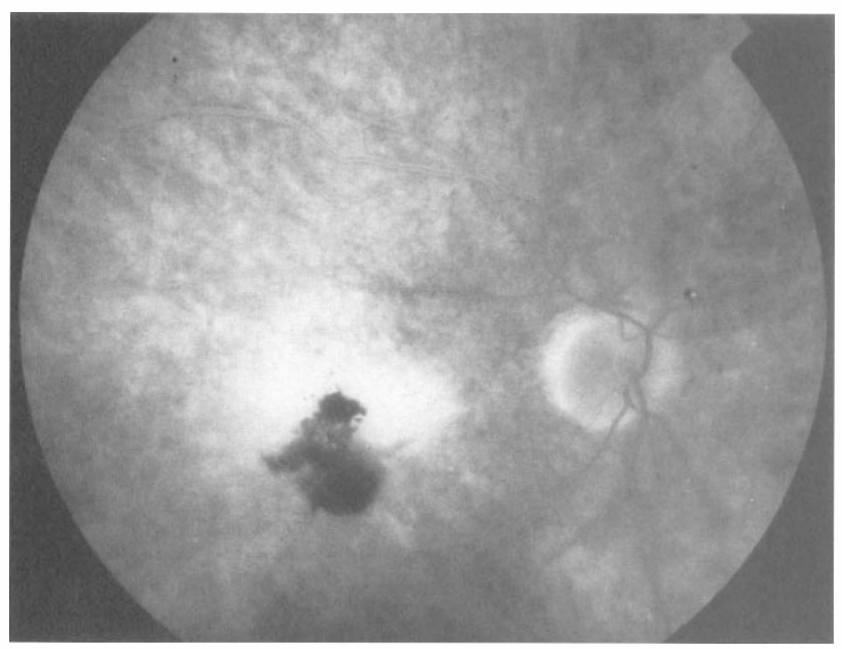

(c)

surgery the patient complained of reduced vision, which was found to have declined to 20/200. Angiography disclosed subfoveal CNV (Fig. 6c). The final visual acuity in that eye remained at 20/200.

\section{DISCUSSION}

All five patients described experienced a sudden progression of the exudative form of AMD during the first year after cataract surgery with IOL implantation, while the maculopathy in the fellow eye remained stable (Table I). Acceleration of the exudative form of AMD has been noted only by a few authors ${ }^{1,9,10}$ although this finding is not universal. ${ }^{11}$ It is often difficult to link cause and effect to an event which occurs following a certain procedure. However, deterioration of retinopathy after cataract surgery has been described for another retinal condition, namely, diabetic retinopathy. ${ }^{2-8}$ The purpose of this study was to call attention to a

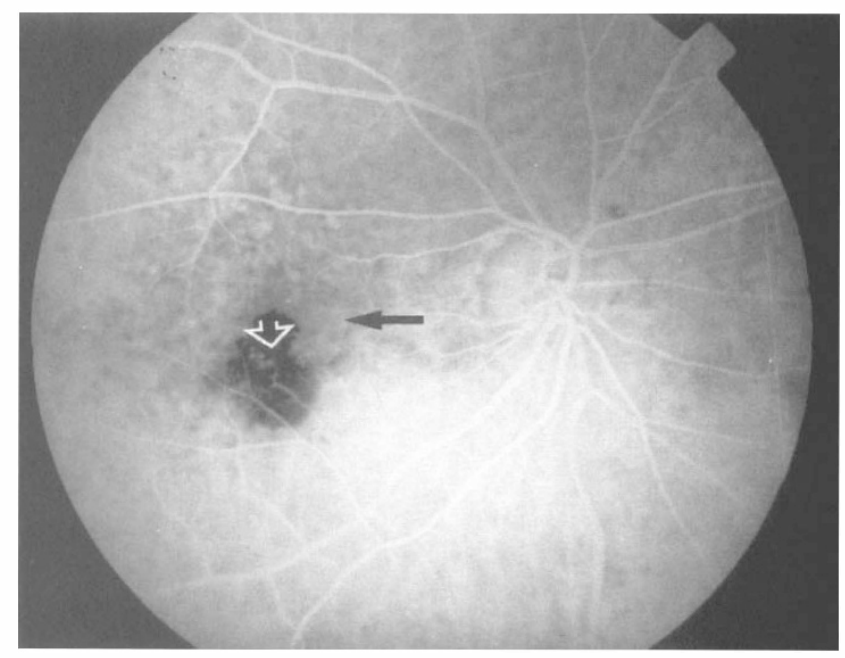

(b)

Fig. 3. Case 2. Post-operative follow-up of the right eye. (a) One month after surgery. Arteriovenous phase shows two foci of blocked fluorescence corresponding to haemorrhages and RPE defects corresponding to drusen. (b) and (c) Four months after surgery. (b) Arteriovenous phase angiogram shows mild diabetic retinopathy with few microaneurysms (white arrow) and CNV (black arrow). (c) Late phase angiogram demonstrates leakage from $C N V$.

possible association between cataract surgery and aggravation of age-related maculopathy.

We selected these patients as examples since we believe each of them represents another aspect of the problems arising when treating patients with AMD and cataract. The first two cases exhibited stable maculopathy for several years prior to cataract surgery but deteriorated shortly afterwards. Two cases (cases 2 and 3) had diabetes mellitus with no diabetic retinopathy pre-operatively. The second case developed diabetic retinopathy post-operatively, concomitant with the exudative AMD. The third case had bilateral cataract and represents the problem of assessing maculopathy in the presence of cataract. By comparing the operated eye with the non-operated eye, it shows that fluorescein angiography can be helpful even when visualisation of the fundus is impaired (Fig. 4). However, in cases where cataract precludes visualisation of the angiogram, an 


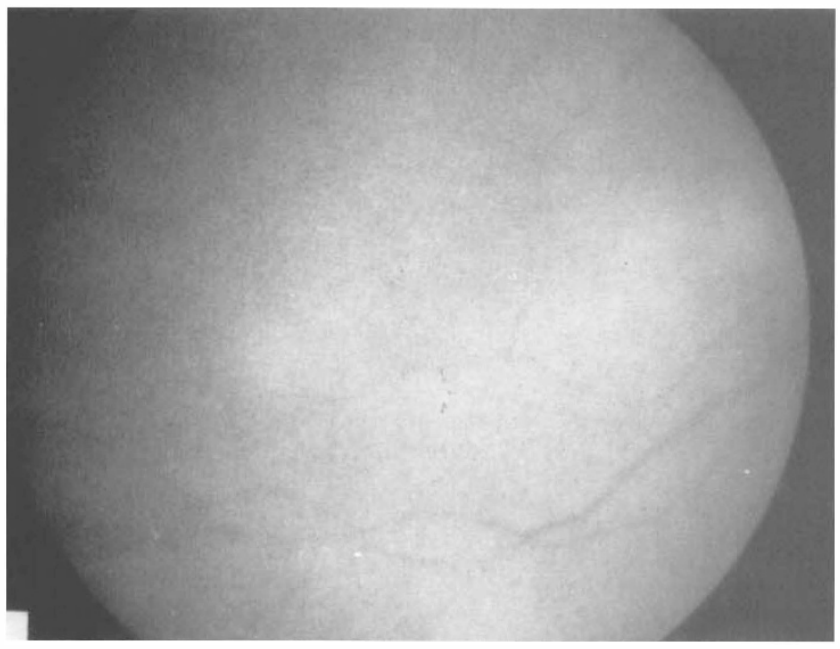

(a)

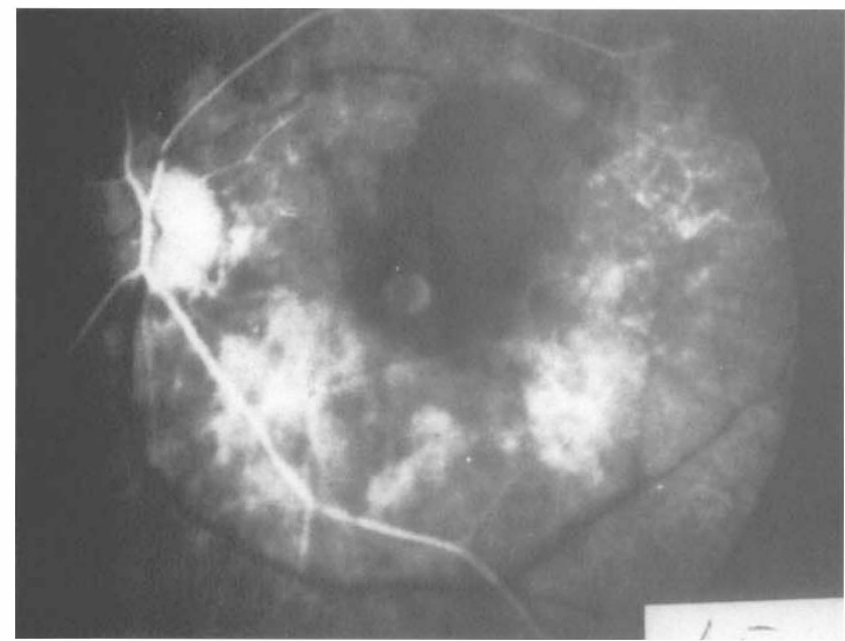

(c)

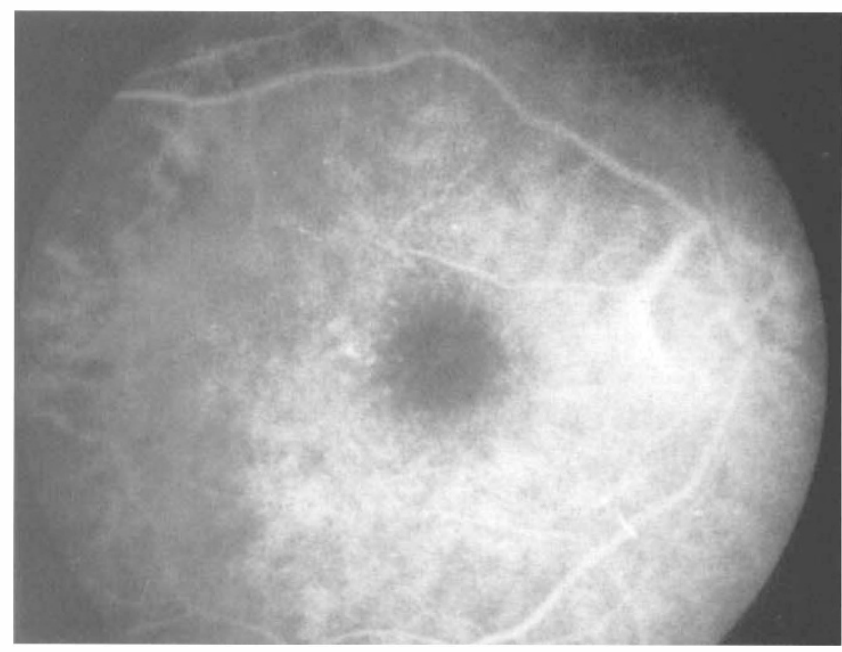

(b)

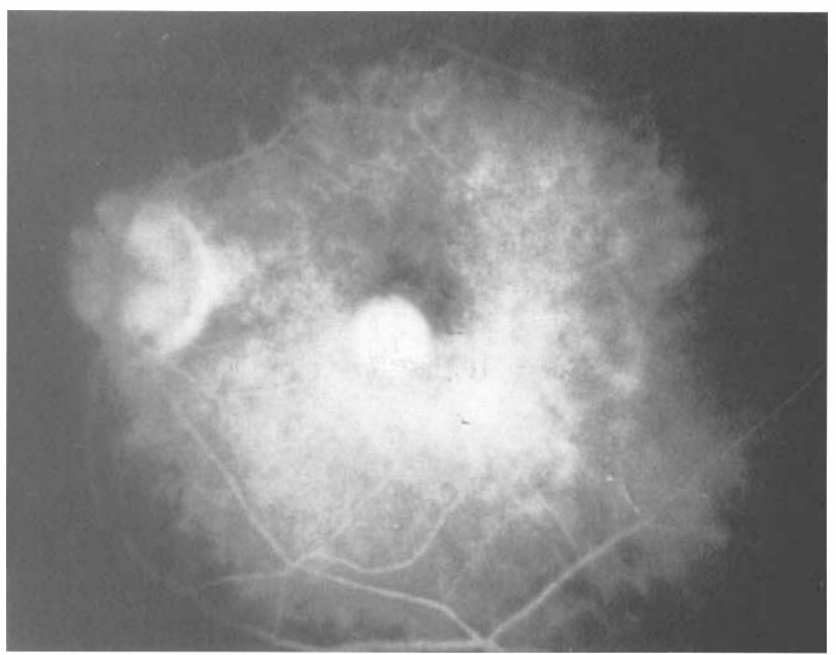

(d)

Fig. 4. Case 3. Seven months after cataract surgery in the left eye. (a) The fundus of the right eye is obscured by the cataract. (b) Angiogram of the right eye discloses RPE defects corresponding to drusen, despite the opacity of the media due to cataract. (c) and (d) Angiograms of the left eye demonstrate leakage from $C N V$.

angiogram can be performed immediately after surgery (cases 1 and 4, Figs. 1 and 5). The cataract may not only obscure maculopathy but also be an obstacle for laser treatment. The last case had advanced AMD in one (non-operated) eye; there- fore the fellow eye was at high risk for development of CNV. Nevertheless, the maculopathy in this highrisk eye remained stable for at least 4 years prior to surgery but worsened shortly afterwards. In the first four cases the preoperative severity of maculopathy

Table I. Ocular findings in operated and non-operated eyes

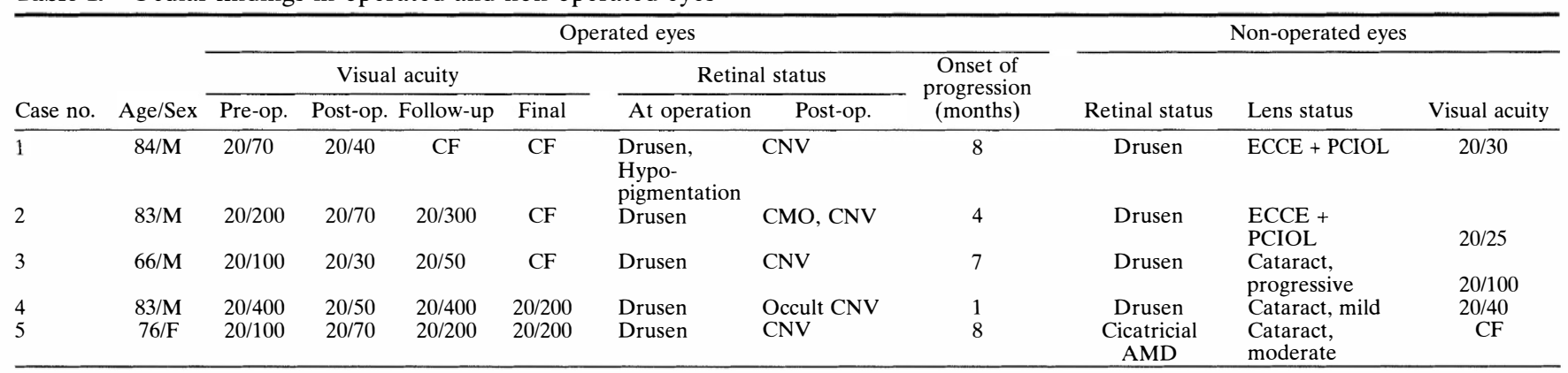

Pre-op., pre-operative; post-op., post-operative; at operation; CNV, choroidal neovascularisation; ECCE + PCIOL, extracapsular cataract extraction with posterior chamber intraocular lens; $\mathrm{CMO}$, cystoid macular oedema; $\mathrm{CF}$, counting fingers; $\mathrm{AMD}$, age-related macular degeneration. 


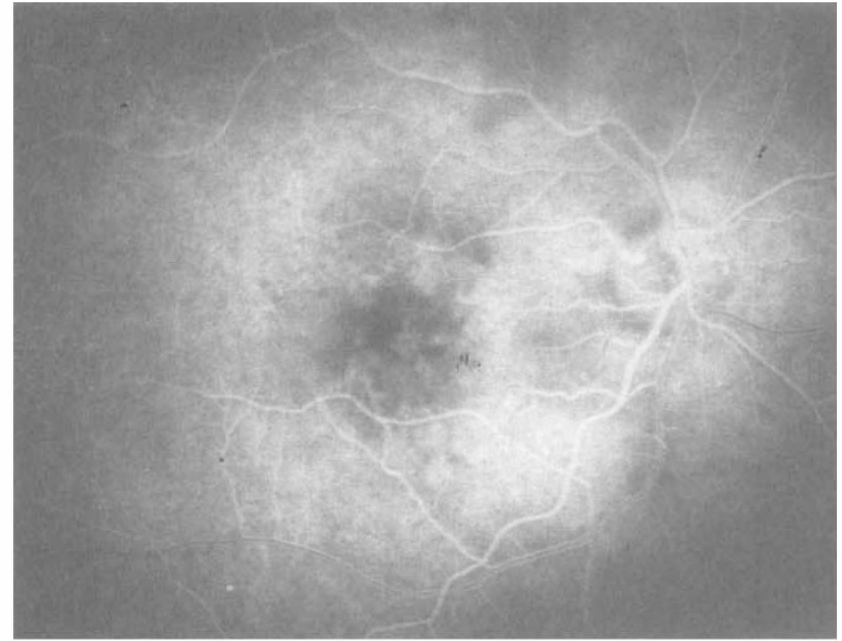

(a)

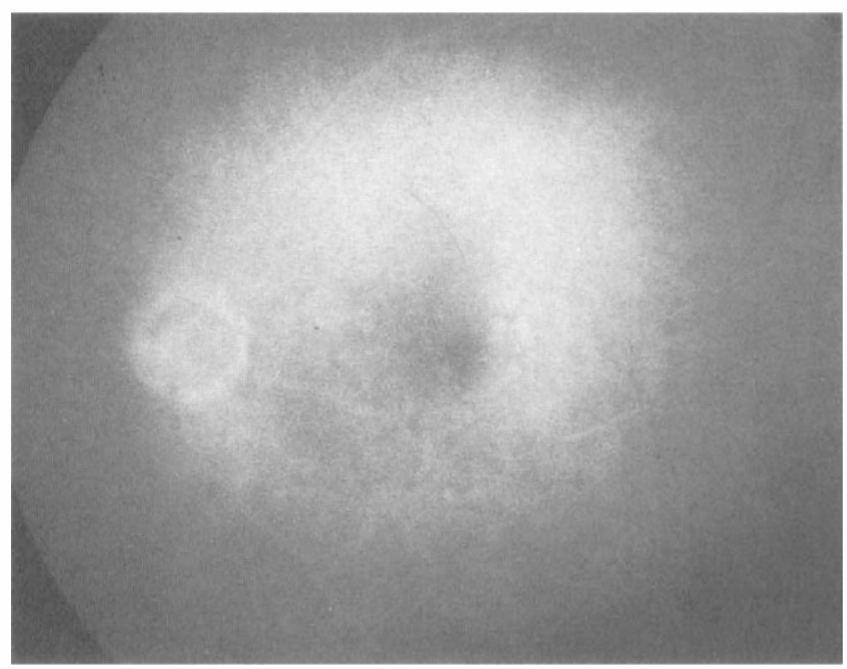

(c)

varied from a few to numerous drusen. However, the macular status appeared similar in both eyes, but subsequently deteriorated in the recently operated eye only (Table I). In two of these patients the fellow eye was phakic and in the other two it was pseudophakic (Table I). Interestingly, although in the latter two patients the post-operative macular status had not changed in the eye that was operated on first, it deteriorated following cataract surgery in the second eye. Thus, at least in cases presented here, an uneventful post-operative course in one eye does not predict a similar one in the fellow eye.

de Jong and associates ${ }^{10}$ had the clinical impression that cataract surgery may promote the growth of new choroidal vessels. In a histological study they found a higher prevalence of disciform scarring in pseudophakic than in phakic eyes. Thus, they suggested that an association between cataract surgery and the progression of AMD may be possible and they offered several explanations to account for it. ${ }^{10}$ Nevertheless, it is not clear how

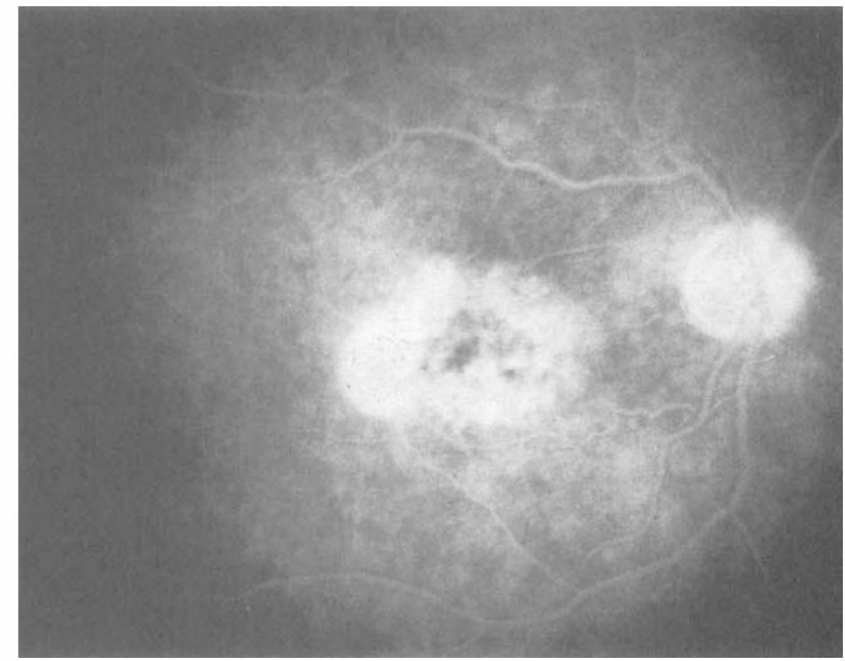

(b)
Fig. 5. Case 4. (a) Angiography 1 week after surgery of the right eye shows RPE defects consistent with drusen. (b) Angiogram 1 month after surgery of the right eye shows $R P E$ detachment with irregular boundaries and nonhomogeneous filling suspected of being occult CNV. (c) Angiogram through a cataract of the left eye shows RPE defects consistent with drusen.

cataract surgery affects macular changes and whether the existing maculopathy is affected by the surgery itself or the removal of the lens or both. With regard to the effect of the lens itself, there are two possible ways in which this may be expressed in patients with AMD. First, as the lens yellows with age it acts increasingly as a barrier, reducing phototoxic injury by filtering out the sun's ultraviolet radiation. Removal of the lens removes this barrier to solar damage. ${ }^{11,12}$ With regard to the effect of the surgery itself, it is possible that the post-operative intraocular inflammation, which is accompanied by the invasion of macrophages known to have angiogenic properties, may induce neovascularisation. ${ }^{13,14}$ This is especially likely to occur in eyes with foci of preexisting pathology such as the drusen and/or alterations in Bruch's membrane seen in AMD. ${ }^{15-21}$ Surgery may also alter the existing equilibrium of the intraocular milieu which might lead to: (1) increased synthesis of the stimulatory substance(s) secreted by the RPE cells ${ }^{22,23}$ and/or (2) reduced 


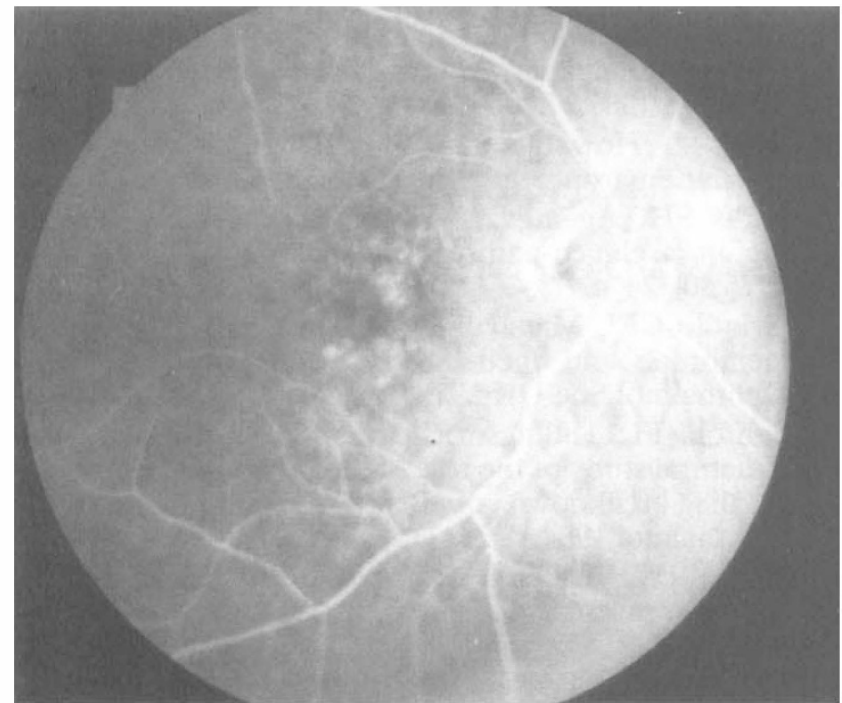

(a)

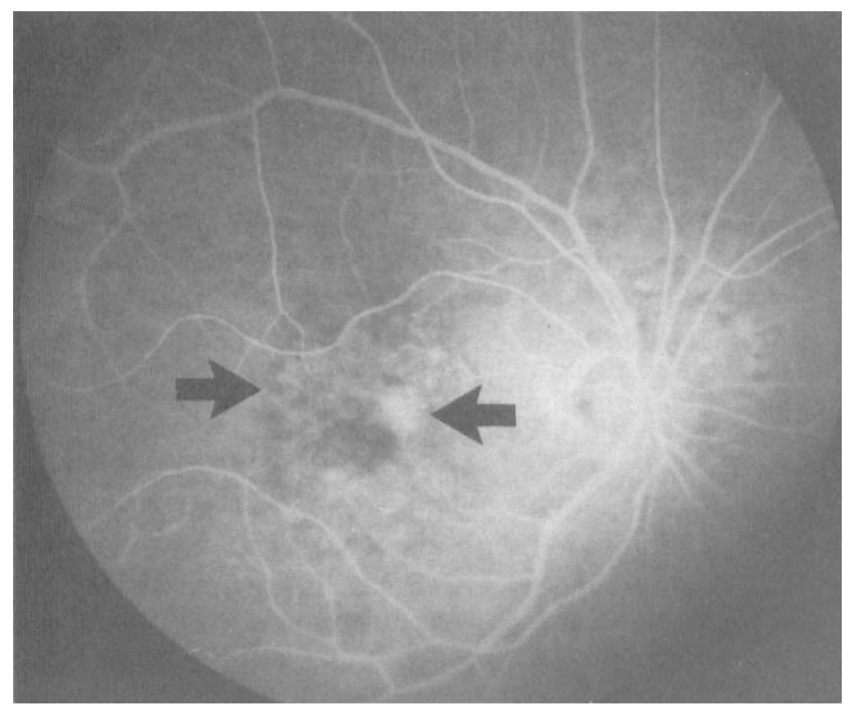

(c)

secretion of vasoinhibitory substances present in the RPE cells. ${ }^{24,25}$ Whatever the trigger may be, the apparent effect is induction of growth of new vessels within as little as a month, as observed by de Jong, ${ }^{10}$ or during the whole first post-operative year. Further investigation is needed: (1) to study the role of each of these factors in the post-operative aggravation of AMD, (2) to determine the post-operative time range over which the development of $\mathrm{CNV}$ is considered to be associated with the surgery and not with the natural course of the disease, and (3) to study the role of other conditions such as diabetes mellitus in the course of maculopathy.

It should be emphasised that many patients with age-related pathology do not experience deterioration of maculopathy following cataract surgery; however, the cases reported here call attention to the possibility of its occurrence and thus increase awareness. These events are especially worth noting

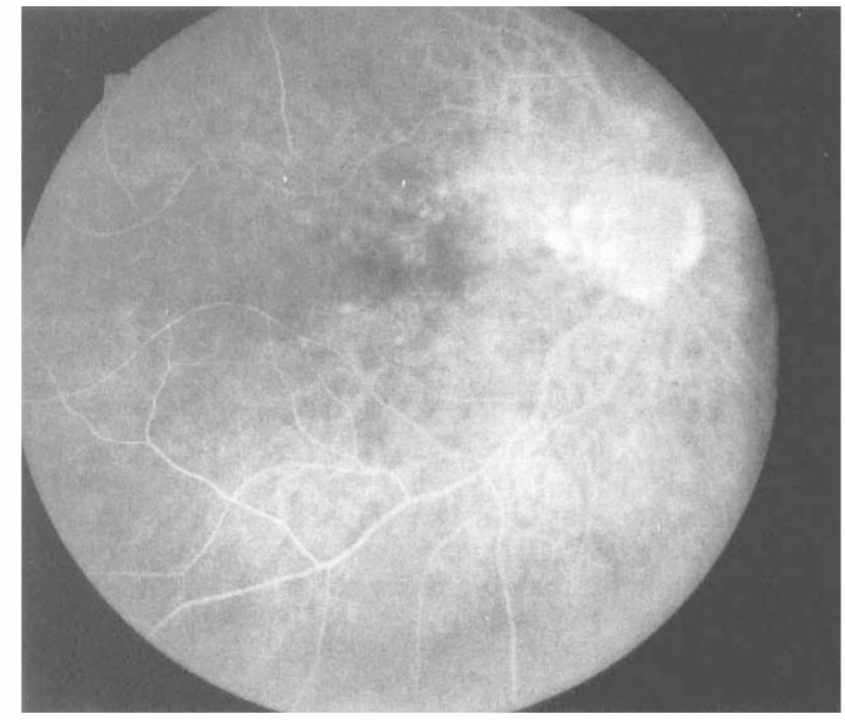

(b)

Fig. 6. Case 5. The pre-and post-operative retinal status of the right eye. (a) and (b) Pre-operative angiography shows in the arteriovenous phase ( 6 minutes), prominent RPE defects consistent with drusen (a) which fade in the late phase (10 minutes, b). (c) Angiogram 8 months after surgery demonstrates subfoveal CNV (arrows).

as cataract is the most common cause of decreased vision in the elderly that can be managed with relative ease and is therefore no longer considered a reason for decreased vision; AMD, on the other hand, is the leading cause of blindness in the Western world. $^{26,27}$ The aim of cataract surgery is to improve the quality of vision, but if the operation results in acceleration of AMD, the opposite may be achieved. A prospective study is required to learn more about the post-operative course of AMD.

For the time being, we recommend a thorough assessment of the retinopathy prior to and after surgery, including fluorescein angiography, and indocyanine green in selected cases. According to the retinal findings, laser treatment should be considered.

The authors wish to thank Zipora Eisner for photographic assistance. 
Presented at the Macula Society Meeting, Palm Beach, Florida, February 1995.

Key words: Age-related macular degeneration, Aggravation, Asymmetric, Extracapsular cataract extraction, Exudative, Intraocular lens implantation.

\section{REFERENCES}

1. Oliver M. Posterior pole changes after cataract extraction in elderly subjects. Am J Ophthalmol 1966;62: 1145-8.

2. Pollack A, Dotan S, Oliver M. The course of diabetic retinopathy following cataract surgery. $\mathrm{Br} \mathrm{J}$ Ophthalmol 1991;75:2-8.

3. Cunliffe IA, Flanagan DW, George NDL, Aggarwaal RJ, Moore AT. Extracapsular cataract surgery with lens implantation in diabetics with and without proliferative retinopathy. Br J Ophthalmol 1991;75: 9-12.

4. Pollack A, Dotan S, Oliver M. Progression of diabetic retinopathy after cataract extraction. $\mathrm{Br} \mathbf{J}$ Ophthalmol 1991;75:547-51.

5. Pollack A, Leiba H, Bukelman A, Abrahami S, Oliver $M$. The course of diabetic retinopathy following cataract surgery in eyes previously treated by laser photocoagulation. Br J Ophthalmol 1992;76:228-31.

6. Pollack A, Leiba H, Bukelman A, Oliver M. Cystoid macular edema following cataract extraction in patients with diabetes. Br J Ophthalmol 1992;76:221-4.

7. Hykin PG, Gregson RMC, Stevens JD, Hamilton PAM. Extracapsular cataract extraction in proliferative diabetic retinopathy. Ophthalmology 1993;100:394-9.

8. Benson WE, Brown GC, Tasman W, McNamara JA, Vander JF. Extracapsular cataract extraction with placement of a posterior chamber lens in patients with diabetic retinopathy. Ophthalmology 1993;100: 730-8.

9. Pollack A, Marcovich A, Bukelman A, Oliver M. Agerelated degeneration after extracapsular cataract extraction with intraocular lens implantation. Ophthalmology 1996;103:1546-1554.

10. Van der Schaft T, Mooy CM, de Bruijn WC, Mulder PGH, Pameyer JH, de Jong PTVM. Increased prevalence of disciform macular degeneration after cataract extraction with implantation of an intraocular lens. Br J Ophthalmol 1994;78:441-5.

11. Cruickshanks KJ, Klein R, Klein BEK. Sunlight and age-related macular degeneration. Arch Ophthalmol 1993;111:514-8.

12. Klein R, Klein BEK, Wang Q, Moss SE. Is age-related maculopathy associated with cataracts? Arch Ophthalmol 1994;112:191-6.
13. Milch FA, Yannuzi LA. Medical and surgical treatment of aphakic cystoid macular edema. Int Ophthalmol Clin 1987;27:205-17.

14. Werb $Z$. How the macrophage regulates the extracellular environment. Am J Anat 1983;166:237.

15. Sarks. SH. Ageing and degeneration in the macular region: a clinico-pathological study. $\mathrm{Br} \mathrm{J}$ Ophthalmol 1976;60:324-41.

16. Grindle CFJ, Marshall J. Ageing changes in Bruch's membrane and their functional implications. Trans Ophthalmol Soc UK 1978;98:172-5.

17. Penfold PL, Killingsworth MC, Sarks SH. An ultrastructural study of the role of leucocytes and fibroblasts in the breakdown of Bruch's membrane. Aust J Ophthalmol 1984;12:23-31.

18. Green RW, McDonnel PJ, Yeo JH. Pathologic features of senile macular degeneration. Ophthalmology 1985; 92:615-27.

19. Penfold PL, Provis JM, Billson FA. Age-related macular degeneration: ultrastructural studies of the relationships of leucocytes to angiogenesis. Graefes Arch Clin Exp Ophthalmol 1987;225:70-6.

20. Young RW. Pathophysiology of age-related macular degeneration. Surv Ophthalmol 1987;31:291-305.

21. Killingsworth MC, Sarks JP, Sarks SH. Macrophages related to Bruch's membrane in age-related macular degeneration. Eye 1990;4:613-21.

22. Mancini MA, Frank RN, Keirn RJ. Does retinal pigment epithelium polarise the choriocapillaries? Invest Ophthalmol Vis Sci 1986;27:336.

23. Morse LA, Sidikaro Y, Terrell J. Retinal pigment epithelium promotes proliferation of choroidal microvessel endothelium in vitro. Invest Ophthalmol Vis Sci 1986;27 (ARVO Suppl):327.

24. Glaser BM, Gampochiaro PA, David JL Jr. Retinal pigment epithelial cells release an inhibitor of neovascularisation. Arch Ophthalmol 1985;103:1870-5.

25. Connor T, Glaser B. RPE cells can simultaneously release inhibitors and stimulators of endothelial cell proliferation. Invest Ophthalmol Vis Sci 1987;28 (ARVO Abstr):203.

26. Ryan SJ. The development of an experimental model of subretinal neovascularisation in disciform macular degeneration. Trans Am Ophthalmol Soc 1979;77: 707-45.

27. Hyman LG. Senile macular degeneration: an epidemiologic case control study [thesis]. Baltimore: Johns Hopkins University, 1981. Quoted by: Fine SL, Murphy RP. Photocoagulation for choroidal neovascularisation. Ophthalmology 1983;90:531-3. 